PROCEEDINGS OF THE

AMERICAN MATHEMATICAL SOCIETY

Volume 131, Number 7, Pages 2261-2269

S 0002-9939(02)06760-6

Article electronically published on October 24, 2002

\title{
GEOMETRIC PROPERTIES CODED IN THE LONG-TIME ASYMPTOTICS FOR THE HEAT EQUATION ON $Z^{n}$
}

\author{
DEBE BEDNARCHAK
}

(Communicated by Jozef Dodziuk)

\begin{abstract}
This paper investigates connections between the long-time asymptotics of heat distribution on a body $\Omega$ in $Z^{n}$, and various geometric properties of $\Omega$, starting from an initially constant heat distribution supported on $\Omega$. We use combinatorial and differential geometric methods. We begin the paper with a result in $R^{n}$.
\end{abstract}

\section{INTRODUCTION}

This paper develops themes from the author's thesis [2], and also owes a considerable debt to the paper of Chavel and Karp [4.

We are interested in the interplay between the evolution of an initial heat distribution on a domain and various aspects of the geometry of the domain. In particular, we are interested in the following problem: Let $\Omega_{1}$ and $\Omega_{2}$ be finite sets in $Z^{n}$, and let $P_{t} 1_{\Omega_{i}}$ be the solution to the heat equation in $Z^{n}$ with initial data $1_{\Omega_{i}}$, where $1_{\Omega_{i}}$ is the indicator function of $\Omega_{i}$. If the long-time asymptotics of $P_{t} 1_{\Omega_{1}}$ and $P_{t} 1_{\Omega_{2}}$ agree on some open set $U$, what geometric properties must $\Omega_{1}$ and $\Omega_{2}$ share? In this paper we investigate this question and the analogous question in $R^{n}$. Our main result, Theorem 4.1 establishes geometric attributes which nearly characterize the informational content of the heat asymptotics when the initial distribution is the indicator function of a finite set in $Z^{n}$. Theorem 2.1 does the same for a relatively compact set in $R^{n}$. Theorem 4.2 is a discrete counterpart of a theorem in [4] which states that given a relatively compact set $\Omega$ and a ball $B$ in $R^{n}$, if $P_{t} 1_{\Omega}$ agrees with $P_{t} 1_{B}$ on an open set $U$ up to order $t^{-(n / 2+1)}$ as $t \rightarrow \infty$, then $\Omega=B$ up to a set of measure 0 .

In 4], Chavel and Karp raise the question of whether this result remains true if one of the sets is only assumed to be convex. We answer this question in the negative in both the continuous and the discrete cases. In more detail, we construct examples of pairs of incongruent convex domains with the same asymptotics. In the discrete case, this construction leads to an interesting diophantine problem.

Received by the editors July 12, 2001 and, in revised form, February 20, 2002.

2000 Mathematics Subject Classification. Primary 58J35, 58J37.

The author was partially supported by the Faculty Scholarship and Development Committee of Long Island University. 


\section{2. $R^{n}$}

We begin with the continuous case as it is more familiar and will provide an introduction to the methods involved. Here, $P_{t} 1_{\Omega_{i}}$ is the solution to the heat equation on $R^{n}$ with initial data $1_{\Omega_{i}}$, the indicator function of the set $\Omega_{i}$. We show

Theorem 2.1. Let $\Omega_{1}$ and $\Omega_{2}$ be relatively compact open sets in $R^{n}$. If

$$
\lim _{t \rightarrow \infty} t^{\frac{n}{2}+1} P_{t}\left(1_{\Omega_{1}}-1_{\Omega_{2}}\right)(x)=0
$$

for $x$ in some open set $U$ in the interior of $R^{n} \backslash\left(\Omega_{1} \cup \Omega_{2}\right)$, then (i) $\Omega_{1}$ and $\Omega_{2}$ have the same volume; (ii) $\Omega_{1}$ and $\Omega_{2}$ have the same center of mass; (iii) $\int_{\Omega_{1}}|y|^{2} d y=$ $\int_{\Omega_{2}}|y|^{2} d y$. Conversely, for relatively compact open sets $\Omega_{1}, \Omega_{2}$, conditions (i), (ii), (iii) imply (2.1) for all $x$ in $R^{n} \backslash\left(\Omega_{1} \cup \Omega_{2}\right)$.

Proof. Following [4] we have

$$
\begin{aligned}
t^{\frac{n}{2}+1} & P_{t}\left(1_{\Omega_{1}}-1_{\Omega_{2}}\right)(x) \\
& =t^{\frac{n}{2}+1}(4 \pi t)^{\frac{-n}{2}} \int_{R^{n}} e^{\frac{-|x-y|^{2}}{4 t}}\left(1_{\Omega_{1}}-1_{\Omega_{2}}\right)(y) d y \\
& =(4 \pi)^{\frac{-n}{2}} t \sum_{k=0}^{\infty} \frac{(-4 t)^{-k}}{k !} \int_{R^{n}}|x-y|^{2 k}\left(1_{\Omega_{1}}-1_{\Omega_{2}}\right)(y) d y \\
& =c t \int_{R^{n}}\left(1_{\Omega_{1}}-1_{\Omega_{2}}\right)(y) d y-\frac{c}{4} \int_{R^{n}}|x-y|^{2}\left(1_{\Omega_{1}}-1_{\Omega_{2}}\right)(y) d y+O\left(t^{-1}\right) .
\end{aligned}
$$

The existence of the limit implies (i) and, therefore,

$$
\int_{R^{n}}|x-y|^{2}\left(1_{\Omega_{1}}-1_{\Omega_{2}}\right)(y) d y=0,
$$

i.e.,

$$
\int_{R^{n}}\left(|x|^{2}-2\langle x, y\rangle+|y|^{2}\right)\left(1_{\Omega_{1}}-1_{\Omega_{2}}\right)(y) d y=0 .
$$

Differentiating with respect to $x$ we have

$$
\sum_{i} \int_{R^{n}}\left(2 x_{i}-2 y_{i}\right)\left(1_{\Omega_{1}}-1_{\Omega_{2}}\right)(y) d y=0
$$

which gives

$$
\sum_{i} \int_{R^{n}} y_{i}\left(1_{\Omega_{1}}-1_{\Omega_{2}}\right)(y) d y=0 .
$$

This is (ii). Substituting into (2.2) we get

$$
\int_{R^{n}}|y|^{2}\left(1_{\Omega_{1}}-1_{\Omega_{2}}\right)(y) d y=0 .
$$

This is (iii).

Conversely, condition (i) makes the first integral in

$$
\text { ct } \int_{R^{n}}\left(1_{\Omega_{1}}-1_{\Omega_{2}}\right)(y) d y-\frac{c}{4} \int_{R^{n}}|x-y|^{2}\left(1_{\Omega_{1}}-1_{\Omega_{2}}\right)(y) d y+O\left(t^{-1}\right)
$$


equal to 0 . The second integral is

$$
\frac{c}{4} \int_{R^{n}}\left(|x|^{2}-2\langle x, y\rangle+|y|^{2}\right)\left(1_{\Omega_{1}}-1_{\Omega_{2}}\right)(y) d y
$$

which is clearly 0 under conditions (i), (ii), and (iii).

We use this result to find pairs of incongruent convex sets for which (2.1) holds. In $R^{n}$, the volume of the ellipsoid

$$
\Omega_{1}=\left\{y: \frac{y_{1}^{2}}{a_{1}^{2}}+\cdots+\frac{y_{n}^{2}}{a_{n}^{2}} \leq 1\right\}
$$

is $c_{1} \prod a_{i}$, its center of mass is the origin, and

$$
\int_{\Omega_{1}} \sum_{j=1}^{n} y_{j}^{2} d y=c_{2}\left(\prod a_{i}\right)\left(a_{1}^{2}+\cdots+a_{n}^{2}\right) .
$$

Here, $c_{1}$ and $c_{2}$ are constants depending on $n$. Any positive solution $\left(m_{1}, \ldots, m_{n}\right)$ to the system

$$
\begin{aligned}
m_{1} m_{2} \cdots m_{n} & =a_{1} a_{2} \cdots a_{n}, \\
m_{1}^{2}+m_{2}^{2}+\cdots+m_{n}^{2} & =a_{1}^{2}+a_{2}^{2}+\cdots+a_{n}^{2},
\end{aligned}
$$

corresponds to an ellipsoid

$$
\Omega_{2}=\left\{y: \frac{y_{1}^{2}}{m_{1}^{2}}+\cdots+\frac{y_{n}^{2}}{m_{n}^{2}} \leq 1\right\}
$$

satisfying conditions (i), (ii) and (iii), and therefore (2.1).

If $\Omega_{1}$ were a ball,

$$
\Omega_{1}=\left\{y: \sum_{j=1}^{n} y_{j}^{2} \leq a^{2}\right\},
$$

and the system would be

$$
\begin{aligned}
m_{1} m_{2} \cdots m_{n} & =a^{n}, \\
m_{1}^{2}+m_{2}^{2}+\cdots+m_{n}^{2} & =n a^{2} .
\end{aligned}
$$

This system has only one positive solution, i.e., $m_{i}=a$. This provides an alternate proof of Theorem 4 of [4], i.e., if $\Omega_{1}$ is a ball, then $\Omega_{2}$ must also be a ball.

\section{THE HEAT KERNEL FOR $Z^{n}$}

For the rest of the paper, we will be dealing with the discrete case. We view $Z^{n}$ as a graph whose edges join a vertex $\left(x_{1}, x_{2}, \ldots, x_{n}\right)$ to its $2 n$ neighbors $\left\{\left(x_{1}, x_{2}, \ldots\right.\right.$, $\left.\left.x_{i} \pm 1, \ldots, x_{n}\right)\right\}, i=1, \ldots, n$. The discrete Laplacian on $Z^{n}$ is defined by

$$
\Delta f(x)=\frac{1}{2 n} \sum_{z \sim x} f(z)-f(x)
$$

where $f$ is a function with finite support and the tilde means that $x$ and $z$ are neighbors.

One can show 2] that the solution to the heat equation $\Delta p(x, y, t)=\frac{\partial}{\partial t} p(x, y, t)$ for a point source at $y$ is

$$
p(x, y, t)=e^{-t} I_{\left(x_{1}-y_{1}\right)}(t / n) \cdots I_{\left(x_{n}-y_{n}\right)}(t / n)
$$


where $I_{r}(t)$ is the modified Bessel function. Indeed, assuming the point source is at the origin, we have

$$
\begin{aligned}
& \Delta_{x} p(x, 0, t) \\
& =\Delta_{x}\left\{e^{-t} I_{x_{1}}(t / n) \cdots I_{x_{n}}(t / n)\right\} \\
& =e^{-t} \frac{1}{2 n} \sum_{z \sim x} I_{z_{1}}(t / n) \cdots I_{z_{n}}(t / n)-e^{-t} I_{x_{1}}(t / n) \cdots I_{x_{n}}(t / n) \\
& =e^{-t} \frac{1}{2 n} \sum_{i=1}^{n}\left\{I_{x_{1}}(t / n) \cdots I_{x_{i}+1}(t / n) \cdots I_{x_{n}}(t / n)\right. \\
& \left.\quad+I_{x_{1}}(t / n) \cdots I_{x_{i}-1}(t / n) \cdots I_{x_{n}}(t / n)\right\} \\
& \quad-e^{-t} I_{x_{1}}(t / n) \cdots I_{x_{n}}(t / n) .
\end{aligned}
$$

The result now follows easily from the standard formula 8]

$$
\frac{\partial}{\partial t} I_{r}(t)=\frac{I_{r+1}(t)+I_{r-1}(t)}{2} .
$$

For a positive real-valued function $\phi$ defined on a finite subset of $Z^{n}$, the solution to the heat equation with initial data $\phi$ is

$$
P_{t} \phi(x)=e^{-t} \sum_{y \in Z^{n}} \phi(y) p(x, y, t) .
$$

\section{4. $Z^{n}$}

In this section we investigate how much geometric information about a domain $\Omega$ is contained in the asymptotics of the heat distribution with initial data $1_{\Omega}$. Theorem 4.1 establishes an equivalence between asymptotic agreement and certain geometric properties.

In the following we will call a set $U$ contained in $Z^{n}$ open if some $x$ in $U$ has $2 n$ neighbors also in $U$. That is, $U$ contains at least one interior point. We have

Theorem 4.1. For two finite sets $\Omega_{1}, \Omega_{2}$ in $Z^{n}, n \geq 2$, if

$$
\lim _{t \rightarrow \infty}(t / n)^{\frac{n}{2}+1} P_{t}\left(1_{\Omega_{1}}-1_{\Omega_{2}}\right)(x)=0
$$

for all $x$ in an open set $U \subset Z^{n} \backslash\left(\Omega_{1} \cup \Omega_{2}\right)$, then

(i) $\Omega_{1}$ and $\Omega_{2}$ contain the same number of points;

(ii) $\sum_{\Omega_{1}} \omega_{i}=\sum_{\Omega_{2}} \omega_{i}$ for each $i, 1 \leq i \leq n$;

(iii) $\sum_{\Omega_{1}} \sum_{i} \omega_{i}^{2}=\sum_{\Omega_{2}} \sum_{i} \omega_{i}^{2}$.

Conversely, if $\Omega_{1}$ and $\Omega_{2}$ are finite sets in $Z^{n}, n \geq 2$, satisfying conditions (i), (ii), (iii), then (4.1) holds for all $x$ in $Z^{n} \backslash\left(\Omega_{1} \cup \Omega_{2}\right)$.

Proof.

$$
\begin{aligned}
& (t / n)^{\frac{n}{2}+1} P_{t}\left(1_{\Omega_{1}}-1_{\Omega_{2}}\right)(x) \\
& \quad=(t / n)^{\frac{n}{2}+1} e^{-t}\left\{\sum_{\omega} I_{\left(\omega_{1}-x_{1}\right)}(t / n) \cdots I_{\left(\omega_{n}-x_{n}\right)}(t / n)\right\}\left(1_{\Omega_{1}}-1_{\Omega_{2}}\right)(\omega) .
\end{aligned}
$$


For large $t$ this is asymptotic to

$$
\begin{aligned}
(t / n)^{\frac{n}{2}+1} e^{-t} \cdot\left(e^{t / n}\right)^{n}\left((2 \pi t / n)^{-1 / 2}\right)^{n} & \cdot \sum_{\omega}\left\{1-\frac{n}{8 t} \sum_{i}\left(4\left(\omega_{i}-x_{i}\right)^{2}-1\right)+O\left(t^{-2}\right)\right\}\left(1_{\Omega_{1}}-1_{\Omega_{2}}\right)(\omega) \\
= & (t / n)\left(\frac{1}{2 \pi}\right)^{n / 2}\left\{\sum_{\omega} 1\left(1_{\Omega_{1}}-1_{\Omega_{2}}\right)\right\} \\
+ & (t / n)\left(\frac{1}{2 \pi}\right)^{n / 2}\left(\frac{n}{8 t}\right)\left\{\sum_{\omega} \sum_{i}\left(4\left(\omega_{i}-x_{i}\right)^{2}-1\right)\right\}\left(1_{\Omega_{2}}-1_{\Omega_{1}}\right)(\omega) .
\end{aligned}
$$

If the limit of this expression as $t \rightarrow \infty$ exists and is equal to 0 , this implies that $\Omega_{1}$ and $\Omega_{2}$ contain the same number of points and therefore

$$
\sum_{\omega}\left\{\sum_{i}\left(\omega_{i}-x_{i}\right)^{2}\left\{1_{\Omega_{1}}-1_{\Omega_{2}}\right\}(\omega)\right\}=0 \text { for all } x \in U .
$$

There is at least one $x$ in $U$ with its neighbors $\left(x_{1}, \ldots, x_{j}+1, \ldots x_{n}\right), 1 \leq j \leq n$, also in $U$. For this $x$ we also have

$$
\sum_{\omega}\left\{\left(2 x_{j}+1-2 \omega_{j}\right)+\sum_{i}\left(\omega_{i}-x_{i}\right)^{2}\right\}\left\{1_{\Omega_{1}}-1_{\Omega_{2}}\right\}(\omega)=0
$$

for each $j, 1 \leq j \leq n$. Combining with (4.2) we have

$$
\sum_{\omega}\left(2 x_{j}+1-2 \omega_{j}\right)\left\{1_{\Omega_{1}}-1_{\Omega_{2}}\right\}(\omega)=0 .
$$

Since $x$ is constant this implies for each $j$

$$
\sum_{\omega} \omega_{j}\left\{1_{\Omega_{1}}-1_{\Omega_{2}}\right\}(\omega)=0
$$

This is (ii).

Furthermore we have

$$
\sum_{\omega} \sum_{j} \omega_{j}\left\{1_{\Omega_{1}}-1_{\Omega_{2}}\right\}(\omega)=0
$$

which implies

$$
\sum_{\omega} \sum_{j} \omega_{j} x_{j}\left(1_{\Omega}-1_{\Omega_{2}}\right)(\omega)=0
$$

for all $x$. Combined with (4.2) this implies

$$
\sum_{\omega}\left\{\sum_{j} \omega_{j}^{2}\left(1_{\Omega_{1}}-1_{\Omega_{2}}\right)(\omega)\right\}=0
$$

This is (iii). 
For the converse, condition (i) implies that the first sum in

$$
\begin{aligned}
& (t / n)\left(\frac{1}{2 \pi}\right)^{n / 2}\left\{\sum_{\omega} 1\left(1_{\Omega_{1}}-1_{\Omega_{2}}\right)(\omega)\right\} \\
& +(t / n)\left(\frac{1}{2 \pi}\right)^{n / 2}\left(\frac{n}{8 t}\right)\left\{\sum_{\omega} \sum_{i}\left(4\left(\omega_{i}-x_{i}\right)^{2}-1\right)\right\}\left(1_{\Omega_{2}}-1_{\Omega_{1}}\right)(\omega)
\end{aligned}
$$

is 0 and that the second sum becomes

$$
c\left\{\sum_{\omega} \sum_{i} \omega_{i}^{2}-\sum_{\omega} \sum_{i} 2 \omega_{i} x_{i}+\sum_{\omega} \sum_{i} x_{i}^{2}\right\}\left(1_{\Omega_{2}}-1_{\Omega_{1}}\right)(\omega) .
$$

The first of these sums is 0 by (iii), the second is 0 by (ii), and the third is 0 by (i).

We note that for sets containing the same number of points, condition (ii) is a discrete analogue of the two sets having the same center of mass.

These geometric properties characterize a particular type of domain in $Z^{n}$, a disk with a Euclidean metric. Using the usual graph metric in $Z^{n}$, i.e., counting edges, the disk with center $c$ and radius $r$ is

$$
B(c ; r)=\left\{x: \sum_{j=1}^{n}\left|x_{j}-c_{j}\right| \leq r\right\} .
$$

The Euclidean-disk with center $c$ and radius $r$ is the set

$$
\tilde{B}(c ; r)=\left\{x: \sum_{j=1}^{n}\left|x_{j}-c_{j}\right|^{2} \leq r^{2}\right\} .
$$

Theorem 4.2. Let $\tilde{B}=\tilde{B}(c ; r)$ be a Euclidean disk in $Z^{n}$ and $\Omega$ a finite set. Suppose

$$
\lim _{t \rightarrow \infty}(t / n)^{\frac{n}{2}+1} P_{t}\left(1_{\Omega}-1_{\tilde{B}}\right)(x)=0
$$

for all $x$ in an open set $U \subset Z^{n} \backslash(\tilde{B} \cup \Omega)$. Then $\Omega=\tilde{B}$.

Proof. From Theorem 4.1 we know that $\Omega$ and $\tilde{B}$ contain the same number of points and satisfy

$$
\sum_{\tilde{B}}\left\{\sum_{i} b_{i}^{2}\right\}=\sum_{\Omega}\left\{\sum_{i} \omega_{i}^{2}\right\} .
$$

Suppose $\Omega \neq \tilde{B}$. Consider the sets $\tilde{B} \backslash \Omega$ and $\Omega \backslash \tilde{B}$. They contain the same number $n$ of points and must satisfy

$$
\sum_{\tilde{B} \backslash \Omega}\left\{\sum_{i} b_{i}^{2}\right\}=\sum_{\Omega \backslash \tilde{B}}\left\{\sum_{i} \omega_{i}^{2}\right\}
$$

(since the same set $\tilde{B} \cap \Omega$ is removed from $\tilde{B}$ and from $\Omega$ ). But $\tilde{B} \backslash \Omega$ contains only points that satisfy $\sum_{i} b_{i}^{2} \leq r^{2}$ and $\Omega \backslash \tilde{B}$ only points that satisfy $\sum_{i} \omega_{i}^{2}>r^{2}$. 
Therefore we have

$$
\begin{aligned}
& \sum_{\tilde{B} \backslash \Omega} \sum_{i} b_{i}^{2} \leq r^{2} \cdot n, \\
& \sum_{\Omega \backslash \tilde{B}} \sum_{i} \omega_{i}^{2}>r^{2} \cdot n,
\end{aligned}
$$

contradicting (4.4).

To see that one must use a Euclidean metric and not the graph metric, consider the following example. In $Z^{2}$, take $B$ to be the graph-metric disk of radius 13 centered at the origin and let

$$
\begin{aligned}
\Omega=B \quad & \{(10,0),(-10,0),(0,10),(0,-10)\} \\
\cup & \{(6,8),(-6,8),(6,-8),(-6,-8)\} .
\end{aligned}
$$

It is straightforward to verify that $B$ and $\Omega$ satisfy conditions (i), (ii), and (iii) of Theorem 4.1 but clearly $\Omega \neq B$. This type of example can easily be produced in higher dimensions.

We turn next to the question of convexity. A set in $Z^{n}$ is convex if it contains all paths of minimal length joining any two elements of the set.

We will show that Theorem 4.2 is not valid for $\Omega_{1}, \Omega_{2}$ even assuming both are convex. We will use Theorem 4.1 to construct pairs of sets in $Z^{n}, n \geq 3$, both convex, satisfying conditions (i), (ii), and (iii) and therefore

$$
\lim _{t \rightarrow \infty}(t / n)^{\frac{n}{2}+1} P_{t}\left(1_{\Omega_{1}}-1_{\Omega_{2}}\right)(x)=0
$$

for all $x$ in $Z^{n} \backslash\left(\Omega_{1} \cup \Omega_{2}\right)$ but with $\Omega_{1} \neq \Omega_{2}$.

Convex sets in $Z^{n}$ are products of intervals, say,

$$
\begin{aligned}
& \Omega_{1}=\left[x_{1}, x_{1}+\left(r_{1}-1\right)\right] \times \cdots \times\left[x_{n}, x_{n}+\left(r_{n}-1\right)\right], \\
& \Omega_{2}=\left[y_{1}, y_{1}+\left(s_{1}-1\right)\right] \times \cdots \times\left[y_{n}, y_{n}+\left(s_{n}-1\right)\right] .
\end{aligned}
$$

We will find conditions on $x_{i}, y_{i}, r_{i}$ and $s_{i}$ to guarantee asymptotic agreement. From condition (i) of Theorem $4.1 \Omega_{1}$ and $\Omega_{2}$ must contain the same number of points, therefore we need $\prod r_{i}=\prod s_{i}$. Condition (ii) says the sum of the $i$ th coordinates over $\Omega_{1}$ is equal to the sum of the $i$ th coordinates over $\Omega_{2}$. The sum of $\omega_{i}$ over $\Omega_{1}$ is

or

$$
\prod_{j \neq i} r_{j} \sum_{k=0}^{r_{i}-1}\left(x_{i}+k\right)=\prod_{j \neq i} r_{j}\left(r_{i} x_{i}+\frac{\left(r_{i}-1\right)\left(r_{i}\right)}{2}\right)
$$

$$
\prod_{j} r_{j}\left(x_{i}+\frac{\left(r_{i}-1\right)}{2}\right) .
$$

Since $\prod r_{i}=\prod s_{i}$, condition (ii) becomes

$$
x_{i}+\frac{\left(r_{i}-1\right)}{2}=y_{i}+\frac{\left(s_{i}-1\right)}{2} \text { for each } i .
$$

Note that this implies that $r_{i}$ and $s_{i}$ must have the same parity. Condition (iii) of Theorem 4.1 says

$$
\sum_{\Omega_{1}} \sum_{i} \omega_{i}^{2}=\sum_{\Omega_{2}} \sum_{i} \omega_{i}^{2}
$$


We have

$$
\begin{aligned}
\sum_{\Omega_{1}} \omega_{i}^{2} & =\prod_{j \neq i} r_{j} \cdot \sum_{k=0}^{r_{i}-1}\left(x_{i}+k\right)^{2} \\
& =\prod_{j \neq i} r_{j} \cdot\left\{r_{i} x_{i}^{2}+2 x_{i} \sum_{k=1}^{r_{i}-1} k+\sum_{k=1}^{r_{i}-1} k^{2}\right\} \\
& =\prod_{j \neq i} r_{j} \cdot\left\{r_{i} x_{i}^{2}+2 x_{i} \frac{\left(r_{i}-1\right) r_{i}}{2}+\frac{\left(r_{i}-1\right) r_{i}\left(2 r_{i}-1\right)}{6}\right\} \\
& =\prod_{j} \cdot\left\{x_{i}^{2}+\left(r_{i}-1\right) x_{i}+\frac{\left(r_{i}-1\right)\left(2 r_{i}-1\right)}{6}\right\} .
\end{aligned}
$$

Therefore (4.7) becomes

$$
\begin{aligned}
\sum_{i} & \left(x_{i}^{2}+x_{i}\left(r_{i}-1\right)+\frac{\left(r_{i}-1\right)\left(2 r_{i}-1\right)}{6}\right) \\
= & \sum_{i}\left(y_{i}^{2}+y_{i}\left(s_{i}-1\right)+\frac{\left(s_{i}-1\right)\left(2 s_{i}-1\right)}{6}\right) .
\end{aligned}
$$

From (4.6) we have

$$
x_{i}^{2}+\left(r_{i}-1\right) x_{i}+\frac{\left(r_{i}-1\right)^{2}}{4}=y_{i}^{2}+\left(s_{i}-1\right) y_{i}+\frac{\left(s_{i}-1\right)^{2}}{4} .
$$

Substituting, we now have

$$
\begin{aligned}
\sum_{i}\left((1 / 6)\left(r_{i}-1\right)\left(2 r_{i}-1\right)-(1 / 4)\left(r_{i}-1\right)^{2}\right) \\
\quad=\sum_{i}\left((1 / 6)\left(s_{i}-1\right)\left(2 s_{i}-1\right)-(1 / 4)\left(s_{i}-1\right)^{2}\right) .
\end{aligned}
$$

This is equivalent to

$$
\sum_{i}\left(r_{i}-1\right)\left(r_{i}+1\right)=\sum_{i}\left(s_{i}-1\right)\left(s_{i}+1\right)
$$

or

$$
\sum_{i} r_{i}^{2}=\sum_{i} s_{i}^{2}
$$

To construct our example we seek two $n$-tuples of integers $\left(r_{1}, \ldots, r_{n}\right),\left(s_{1}, \ldots, s_{n}\right)$ with equal products, equal square sums and matching parities. The $x_{i}$ are arbitrary and the $y_{i}$ are chosen according to equation (4.6). One solution is to take for $\left(s_{1}, \ldots, s_{n}\right)$ an allowable permutation of $\left(r_{1}, \ldots, r_{n}\right)$. In that case, $\Omega_{2}$ is a rotation of $\Omega_{1}$. There are also nontrivial solutions. See, for example, [6], [3], and [7] for solutions in $Z^{3}$. Solutions for higher dimensions may be obtained from these.

Note that in $Z^{2}$ we would want pairs of positive integers $r_{1}, r_{2}$ and $s_{1}, s_{2}$ such that $r_{1} r_{2}=s_{1} s_{2}$ and $r_{1}^{2}+r_{2}^{2}=s_{1}^{2}+s_{2}^{2}$. If $r_{1}$ and $r_{2}$ are given, then $s_{1}$ and $s_{2}$ are determined; either $s_{1}=r_{1}$ and $s_{2}=r_{2}$, and $\Omega_{2}=\Omega_{1}$ or $s_{1}=r_{2}$ and $s_{2}=r_{1}$, and $\Omega_{2}$ is a rotation of $\Omega_{1}$ 


\section{REFERENCES}

[1] E.J. Barbeau, Power Play, Mathematical Association of America, Washington, D.C., 1997. MR 98d:11001

[2] D. Bednarchak, Heat diffusion on graphs, Ph.D. Thesis, CUNY Graduate School, 1994.

[3] E.T. Bell, Reciprocal arrays and Diophantine analysis, Amer. J. Math 55 (1933), 50-66.

[4] I. Chavel and L. Karp, Movement of hot spots on Riemannian manifolds, J. d'Analyse Math. 55 (1990), 271-286. MR 92b:58223

[5] A. Gray and G.B. Mathews, A Treatise on Bessel Functions and their Applications to Physics, Dover, New York, 1966. MR 33:1506 table errata MR 56:703 table errata MR 57:16739

[6] J.B. Kelly, Two equal sums of three squares with equal products, Amer. Math. Monthly 98 (1991), 527-529. MR 92j:11025

[7] L.J. Lander, Geometric aspects of Diophantine equations involving equal sums of like powers, Amer. Math. Monthly 75 (1968), 1061-1073. MR 38:5710

[8] G.N. Watson, A Treatise on the Theory of Bessel Functions, Cambridge University Press, London, 1962. MR 6:64a; reprint MR 96i:33010

Department of Mathematics, Long Island University, 1 University Plaza, Brooklyn, NEW YORK 11201-8423

E-mail address: dbednarc@liu.edu 